This is a self-archived version of an original article. This version may differ from the original in pagination and typographic details.

Author(s): Hayen, L.; Kostensalo, Joel; Severijns, N.; Suhonen, Jouni

Title: First-forbidden transitions in reactor antineutrino spectra

Year: 2019

Version: Published version

Copyright: @ 2019 American Physical Society

Rights: In Copyright

Rights url: http://rightsstatements.org/page//nC/1.0/?language=en

Please cite the original version:

Hayen, L., Kostensalo, J., Severijns, N., \& Suhonen, J. (2019). First-forbidden transitions in reactor antineutrino spectra. Physical Review C, 99(3), Article 031301(R).

https://doi.org/10.1103/physrevc.99.031301 


\title{
First-forbidden transitions in reactor antineutrino spectra
}

\author{
L. Hayen, ${ }^{1,}{ }^{*}$ J. Kostensalo, ${ }^{2}$ N. Severijns, ${ }^{1}$ and J. Suhonen ${ }^{2}$ \\ ${ }^{1}$ Instituut voor Kern- en Stralingsfysica, KU Leuven, Celestijnenlaan 200D, B-3001 Leuven, Belgium \\ ${ }^{2}$ Department of Physics, University of Jyväskylä, P.O. Box 35, FI-40014 University of Jyväskylä, Finland
}

(Received 31 May 2018; revised manuscript received 21 December 2018; published 6 March 2019)

\begin{abstract}
We study the dominant forbidden transitions in the antineutrino spectra of the fission actinides from $4 \mathrm{MeV}$ onward using the nuclear shell model. Through explicit calculation of the shape factor, we show the expected changes in cumulative electron and antineutrino spectra. Relative to the allowed approximation this results in a minor decrease of electron spectra above $4 \mathrm{MeV}$, whereas an increase of several percent is observed in antineutrino spectra. We show that forbidden transitions dominate the spectral flux for most of the experimentally accessible range. Based on the shell model calculations we attempt a parametrization of forbidden transitions and propose a spectral correction for all first-forbidden transitions. We enforce correspondence with the Institut Laue-Langevin data set using a summation+conversion approach. When compared against modern reactor neutrino experiments, the resultant spectral change is observed to be of comparable magnitude and shape as the reported spectral shoulder.
\end{abstract}

DOI: 10.1103/PhysRevC.99.031301

For the past years, neutrino physics has seen a flurry of interest in the so-called reactor anomaly [1-3], a $6 \%$ deficit in the experimentally observed antineutrino count rate relative to theoretical predictions. Together with more long-standing anomalies (reported by LSND [4,5] and GALLEX [6] collaborations), much theoretical interest has gone toward the possibility of one or more eV-scale sterile neutrinos [7,8]. Motivated by these findings, several dedicated experiments worldwide are directly looking for an oscillation pattern at very short baselines, meaning results are independent of theoretical calculations. The explored parameter space is, however, inspired by theoretical estimates. Many experiments are in the process of data taking and have published preliminary results [9-14]. So far, all of these exclude the best theoretical fit value [8] with $\geqslant 2 \sigma$, so a better understanding and control of the theoretical estimate remains an important goal.

With the availability of precision antineutrino spectra, all modern long baseline reactor neutrino experiments have additionally observed a spectral disagreement with respect to theoretical predictions between 4 and $6 \mathrm{MeV}$ [15-17]. Up to now, this so-called shoulder has remained unexplained, and several possibilities have been proposed for its solution [18-22]. The role of (first-)forbidden transitions in both the anomaly and shoulder has so far received limited study, either in parametrized [23] or microscopic treatments [24]. Based on the behavior of pseudoscalar $\left(\Delta J^{\pi}=0^{-}\right)$transitions the forbidden influence has been estimated as negligible [20], despite their flux dominance in the region of interest [25]. Here we investigate the influence of first-forbidden $\beta$ decays by calculating the shape factor of the dominant transitions in

*leendert.hayen@kuleuven.be the region of interest using the nuclear shell model, and show its far-reaching consequences.

We use the formalism of Behrens and Bühring [26] to describe the spectral shape, taking into account finite-size and Coulomb corrections at all levels. We write the $\beta$ spectrum as

$$
\frac{d N}{d W}=p W\left(W-W_{0}\right)^{2} F(Z, W) C(Z, W) K(Z, W),
$$

where $W=E / m_{e} c^{2}+1$ is the total $\beta$ energy, $p=\sqrt{W^{2}-1}$ the momentum, $W_{0}$ the spectral endpoint, and $Z$ the proton number of the daughter. Additionally, $F(Z, W)$ is the Fermi function, $C(Z, W)$ the shape factor, and $K(Z, W)$ additional higher-order effects [27]. In previous analyses [3,28] forbidden transitions were approximated as allowed, either using $C=1$ or including a linear weak magnetism correction so that $d C / d W=0.67 \% \mathrm{MeV}^{-1}$. In the so-called Huber-Mueller (H-M) case, all forbidden transitions were assumed to have a unique shape [2]. We compare our findings against the allowed approximation, and comment on the H-M approximation.

We write the generalized unique forbidden shape factor of order $L$ as [29]

$$
C_{U}=\sum_{k=1}^{L} \lambda_{k} \frac{p^{2(k-1)} q^{2(L-k)}}{(2 k-1) ![2(L-k)+1] !},
$$

and for illustrative purposes we write the first-forbidden pseudoscalar and pseudovector shape factors using their dominant parts as

$$
\begin{aligned}
& C_{0^{-}}=1+\frac{2 R}{3 W} b+\mathcal{O}\left(\alpha Z R, W_{0} R^{2}\right), \\
& C_{1^{-}}=1+a W+\mu_{1} \gamma \frac{b}{W}+c W^{2},
\end{aligned}
$$


TABLE I. Dominant forbidden transitions above $4 \mathrm{MeV}$. Here $Q_{\beta}$ is the ground-state to ground-state $Q$ value, $E_{\mathrm{ex}}$ the excitation energy of the daughter level, BR the branching ratio of the transition normalized to one decay, and FY the cumulative fission yield of ${ }^{235} \mathrm{U}$ from the ENDF database [48].

\begin{tabular}{lcccccc}
\hline \hline $\begin{array}{l}{ }^{\mathrm{Nuclide}} \\
{ }^{2}\end{array}$ & $\begin{array}{c}Q_{\beta} \\
(\mathrm{MeV})\end{array}$ & $\begin{array}{c}E_{\text {ex }} \\
(\mathrm{MeV})\end{array}$ & $\begin{array}{c}\mathrm{BR} \\
(\%)\end{array}$ & $J_{i}^{\pi} \rightarrow J_{f}^{\pi}$ & $\mathrm{FY}$ & $\Delta J$ \\
$(\%)$ & \\
\hline${ }^{89} \mathrm{Br}$ & 8.3 & 0 & 16 & $3 / 2^{-} \rightarrow 3 / 2^{+}$ & 1.1 & 0 \\
${ }^{90} \mathrm{Rb}$ & 6.6 & 0 & 33 & $0^{-} \rightarrow 0^{+}$ & 4.5 & 0 \\
${ }^{91} \mathrm{Kr}$ & 6.8 & 0.11 & 18 & $5 / 2^{+} \rightarrow 5 / 2^{-}$ & 3.5 & 0 \\
${ }^{92} \mathrm{Rb}$ & 8.1 & 0 & 95.2 & $0^{-} \rightarrow 0^{+}$ & 4.8 & 0 \\
${ }^{93} \mathrm{Rb}$ & 7.5 & 0 & 35 & $5 / 2^{-} \rightarrow 5 / 2^{+}$ & 3.5 & 0 \\
${ }^{94} \mathrm{Y}$ & 4.9 & 0.92 & 39.6 & $2^{-} \rightarrow 2^{+}$ & 6.5 & 0 \\
${ }^{95} \mathrm{Sr}$ & 6.1 & 0 & 56 & $1 / 2^{+} \rightarrow 1 / 2^{-}$ & 5.3 & 0 \\
${ }^{96} \mathrm{Y}$ & 7.1 & 0 & 95.5 & $0^{-} \rightarrow 0^{+}$ & 6.0 & 0 \\
${ }^{97} \mathrm{Y}$ & 6.8 & 0 & 40 & $1 / 2^{-} \rightarrow 1 / 2^{+}$ & 4.9 & 0 \\
${ }^{98} \mathrm{Y}$ & 9.0 & 0 & 18 & $0^{-} \rightarrow 0^{+}$ & 1.9 & 0 \\
${ }^{133} \mathrm{Sn}$ & 8.0 & 0 & 85 & $7 / 2^{-} \rightarrow 7 / 2^{+}$ & 0.1 & 0 \\
${ }^{135} \mathrm{Te}$ & 5.9 & 0 & 62 & $(7 / 2-) \rightarrow 7 / 2^{+}$ & 3.3 & 0 \\
${ }^{136 m} \mathrm{I}$ & 7.5 & 1.89 & 71 & $\left(6^{-}\right) \rightarrow 6^{+}$ & 1.3 & 0 \\
${ }^{136 m} \mathrm{I}$ & 7.5 & 2.26 & 13.4 & $\left(6^{-}\right) \rightarrow 6^{+}$ & 1.3 & 0 \\
${ }^{137} \mathrm{I}$ & 6.0 & 0 & 45.2 & $7 / 2^{+} \rightarrow 7 / 2^{-}$ & 3.1 & 0 \\
${ }^{142} \mathrm{Cs}$ & 7.3 & 0 & 56 & $0^{-} \rightarrow 0^{+}$ & 2.7 & 0 \\
${ }^{86} \mathrm{Br}$ & 7.3 & 0 & 15 & $\left(1^{-}\right) \rightarrow 0^{+}$ & 1.6 & 1 \\
${ }^{86} \mathrm{Br}$ & 7.3 & 1.6 & 13 & $\left(1^{-}\right) \rightarrow 2^{+}$ & 1.6 & 1 \\
${ }^{87} \mathrm{Se}$ & 7.5 & 0 & 32 & $3 / 2^{+} \rightarrow 5 / 2^{-}$ & 0.8 & 1 \\
${ }^{89} \mathrm{Br}$ & 8.3 & 0.03 & 16 & $3 / 2^{-} \rightarrow 5 / 2^{+}$ & 1.1 & 1 \\
${ }^{91} \mathrm{Kr}$ & 6.8 & 0 & 9 & $5 / 2^{+} \rightarrow 3 / 2^{-}$ & 3.4 & 1 \\
${ }^{134 m} \mathrm{Sb}$ & 8.5 & 1.69 & 42 & $(7-) \rightarrow 6^{+}$ & 0.8 & 1 \\
${ }^{134 m} \mathrm{Sb}$ & 8.5 & 2.40 & 54 & $\left(7^{-}\right) \rightarrow\left(6^{+}\right)$ & 0.8 & 1 \\
${ }^{138} \mathrm{I}$ & 8.0 & 0 & 26 & $(1-) \rightarrow 0^{+}$ & 1.5 & 1 \\
${ }^{140} \mathrm{Cs}$ & 6.2 & 0 & 36 & $1^{-} \rightarrow 0^{+}$ & 5.7 & 1 \\
${ }^{88} \mathrm{Rb}$ & 5.3 & 0 & 76.5 & $2^{-} \rightarrow 0^{+}$ & 3.6 & 2 \\
${ }^{94} \mathrm{Y}$ & 4.9 & 0 & 41 & $2^{-} \rightarrow 0^{+}$ & 6.5 & 2 \\
${ }^{95} \mathrm{Rb}$ & 9.2 & 0 & 0.1 & $5 / 2^{-} \rightarrow 1 / 2^{+}$ & 0.8 & 2 \\
${ }^{139} \mathrm{Xe}$ & 5.1 & 0 & 15 & $3 / 2^{-} \rightarrow 7 / 2^{+}$ & 5.0 & 2 \\
\hline \hline
\end{tabular}

where $R$ is the nuclear radius, $\alpha$ is the fine-structure constant, $\gamma=\sqrt{1-(\alpha Z)^{2}}, q=W_{0}-W$ is the (anti)neutrino momentum, and $\lambda_{k}$ is a Coulomb correction function. Here $a, b$, and $c$ are (complex) combinations of nuclear matrix elements, corresponding to powers of $W+1,-1$, and +2 . Note that we have not used the simplified expressions of Eqs. (3) and (4), but rather used the complete formulation as can be found, e.g., in Ref. [30]. As such, we additionally take into account finite-size effects and Coulomb corrections to the nuclear matrix elements. As cancellations can occur in the main matrix elements, the latter can eventually dominate the shape factor. The importance of these corrections cannot be understated, in particular for the high masses of the actinide fission fragments [31].

Based on the compilation in Ref. [25] we have selected 29 forbidden transitions, listed in Table I. All transitions have a $\beta$ spectrum endpoint above $4 \mathrm{MeV}$, meaning that all contribute to the observed spectral shoulder. Using the experimental results obtained at the Institut Laue-Langevin (ILL) [32-36], the selected transitions correspond to at least $50 \%$ of the

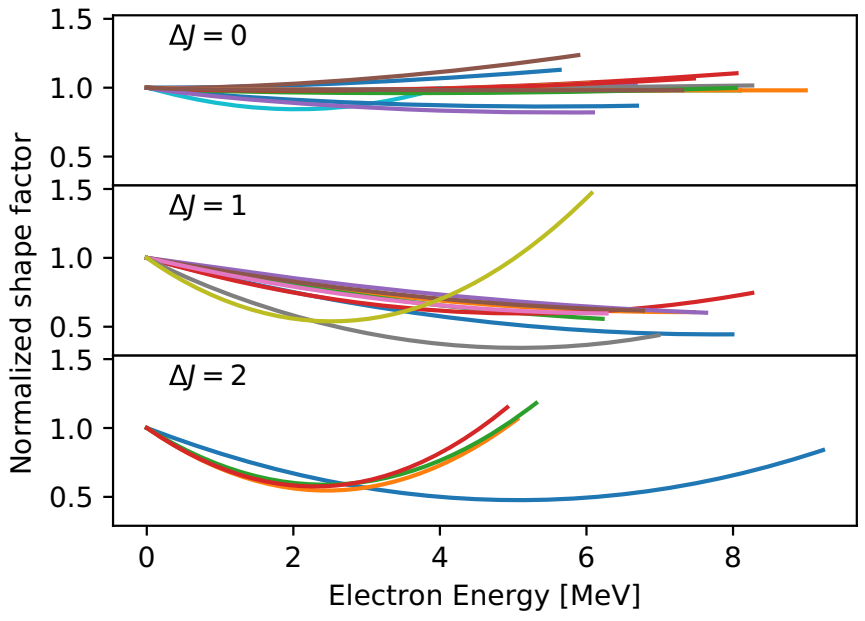

FIG. 1. Calculated shape factors $C$ vs electron kinetic energy, categorized according to the spin-parity change of the transition. For allowed transitions $C \approx 1$. Each shape factor was normalized to its value at $E=0$. Results correspond to $g_{A}=0.9$ and $\epsilon_{\mathrm{MEC}}=$ 1.4, where applicable $[31,42]$. Two cases stand out: ${ }^{94} \mathrm{Y}\left(2^{-} \rightarrow 2^{+}\right)$ and ${ }^{86} \mathrm{Br}\left(1^{-} \rightarrow 2^{+}\right)$. Both contain strong admixtures of $\Delta J=2$ operators, since both $2^{+}$final states identify as vibrational excitations of the $0^{+}$ground state.

cumulative electron flux in the region of interest $(2-8 \mathrm{MeV})$, and exceed $65 \%$ at $6 \mathrm{MeV}$ for all fission actinides [31]. The shell model calculations were performed using the shell model code NUSHELLX@MSU [37]. For nuclei with $A<100$ the effective interaction glepn [38] was adopted in a full model space consisting of the proton orbitals $0 f_{5 / 2}-1 p-0 g_{9 / 2}$ and the neutron orbitals $1 d-2 s$. The ${ }^{86} \mathrm{Br}$ and ${ }^{89} \mathrm{Br}$ cases were calculated using the interaction jj45pna $[39,40]$, in the full model space spanned by the proton orbitals $0 f_{5 / 2}-1 p-0 g_{9 / 2}$ and the neutron orbitals $0 g_{7 / 2}-2 s-1 d-0 h_{11 / 2}$. For the nuclei with $A=133-142$ the Hamiltonian jj56pnb [41] was used in the full model space spanned by the proton orbitals $0 g_{7 / 2}-1 d-2 s-0 h_{11 / 2}$ and neutron orbitals $0 h_{9 / 2}-1 f-2 p-0 i_{13 / 2}$ for $A<139$, while for the heavier nuclei the proton orbital $0 h_{11 / 2}$ and the neutron orbital $0 i_{13 / 2}$ were kept empty due to the enormous dimensions of a full model space calculation. Uncertainties due to $g_{A}$ quenching and meson exchange currents (MECs) in pseudoscalar transitions in the fission fragment region have been reported [31,42-46], and will be briefly commented upon further in this work.

We have separately used the ENSDF [47] and ENDF [48] decay libraries. While the former suffers from multiple cases of the pandemonium effect [49], the latter has been corrected to obtain improved agreement with experimental reactor data [50,51]. Unless mentioned explicitly, the results below are obtained using the ENDF/B-VIII.0 decay library with spin-parity information from ENSDF. Transitions with unknown spin change are assumed allowed and unknown branching ratios are distributed equally from the remaining intensity [31].

Figure 1 shows the calculated shape factors categorized according to the change in spin-parity. Clearly almost all shape factors deviate significantly from unity. The spin change 


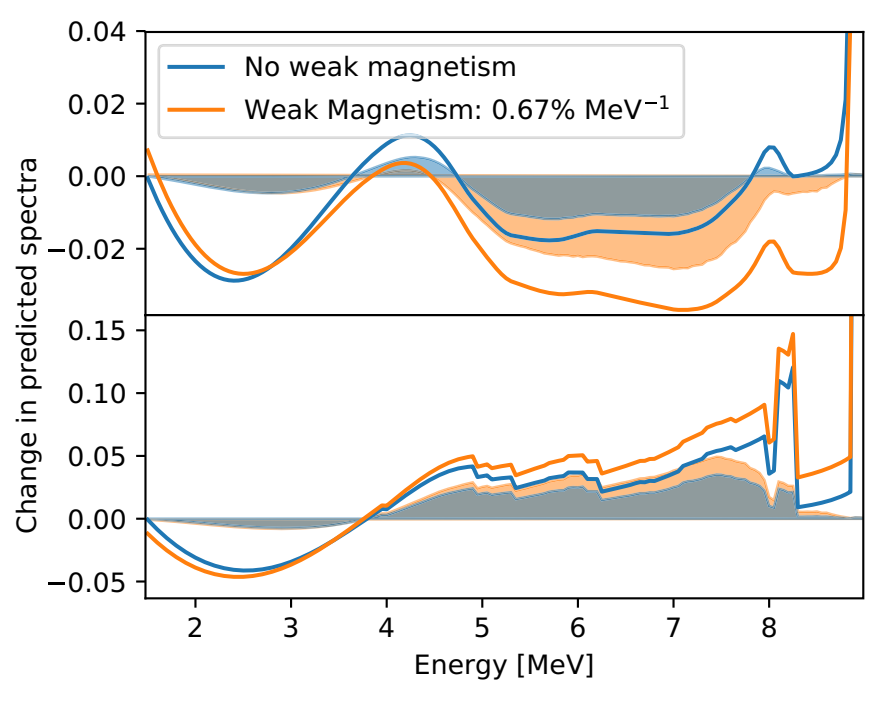

FIG. 2. Top panel: Change in the predicted electron spectra of the considered transitions compared to the allowed approximation with an optional weak magnetism correction. Bottom panel: Change in the predicted antineutrino spectrum compared to the allowed approximation. Shaded areas correspond to the results multiplied by the total spectral contribution compared to experimental flux results. The energy axis refers to the kinetic energy of the electron (top) and antineutrino (bottom).

is a good predictor of the calculated shape factor, with the exception of pseudoscalar, $\Delta J^{\pi}=0^{-}$, transitions. From Eq. (3) its behavior should be trivial with $|b R| \sim 10^{-2}$, yet large variations appear. Many of these transitions connect initial and final states with spins larger than zero, meaning additional $\Delta J=1,2$ operators contribute non-negligibly. As such, in many cases the energy dependence is dominated by higherorder operators. Even though results appear to scatter around unity, the limited number of contributing branches forbids simple statistical averaging arguments.

Using the fission yields of the ENDF database [48], Fig. 2 shows the change of both electron and antineutrino spectra compared to the allowed approximation with an optional weak magnetism correction. Compared to the weak magnetism correction typically used [2,3], electron spectra see a modest $2 \%$ decrease in the 4 to $8 \mathrm{MeV}$ region. Cumulative antineutrino spectra, on the other hand, see a change of up to $5 \%$ in the same region. The parabolic behavior below $4 \mathrm{MeV}$ is almost entirely attributable to first-forbidden unique decays (see Fig. 1).

While a significant fraction of the spectral change occurs because of pseudovector $\left(\Delta J^{\pi}=1^{-}\right)$transitions, inspection of Fig. 1 should make it clear that even pseudoscalar transitions carry significant deviations from unity. Previous arguments for its neglect [20] have used ${ }^{92} \mathrm{Rb}$ and ${ }^{96} \mathrm{Y}$ as examples for their predictions, even though many important pseudoscalar transitions are not pure $0^{-} \rightarrow 0^{+}$decays (see Fig. 1).

The results presented in Fig. 2 depend on the value used for the weak magnetism correction in the allowed approximation. In the formalism by Holstein [52] its electron spectral dependence is written as

$$
\left(\frac{d N}{d W}\right)^{\mathrm{wm}} \propto \frac{4 b}{3 M c}\left(W-\frac{W_{0}}{2}-\frac{1}{2 W}\right),
$$

where $b$ and $c$ are the weak magnetism and Gamow-Teller form factors, respectively, and $M$ is the nuclear mass, omitting phase-space factors and additional correction factors [27]. Above a few $\mathrm{MeV}$ then, its energy dependence is approximately linear leading to the behavior observed in Fig. 2. Evaluation of this ratio of form factors can happen through a combination of the conserved vector current hypothesis (rendering $b$ ) and the $f t$ value (rendering $c$ ), or having to rely on many-particle quantum calculations such as the nuclear shell model. The former is only possible for decays occurring within isospin multiplets and is limited to nuclei where $N \sim$ $Z$ [53]. Previous analyses have taken this approach [3] and used results extracted from mass $A \leqslant 28$ systems with the assumption that they are equally valid at high masses, leading to $d C / d W=0.67 \% \mathrm{MeV}^{-1}$ quoted above [2,3]. When one cannot rely on symmetries, one must attempt to calculate these by introducing the impulse approximation. Here, the nuclear current is treated as a sum of noninteracting nucleon currents and the form factor ratio reduces to

$$
\frac{b}{A c}=\frac{1}{g_{A}}\left(g_{M}+g_{V} \frac{\mathcal{M}_{L}}{\mathcal{M}_{\mathrm{GT}}}\right)
$$

where $g_{M}=4.706$ and $\mathcal{M}_{L}, \mathcal{M}_{\mathrm{GT}}$ are the orbital and GamowTeller matrix elements [52]. An extensive study [54] performed in the fission fragment region found large variations in $\mathcal{M}_{L} / \mathcal{M}_{\mathrm{GT}}$ compared to simple approximations, but concluded that the latter is sufficiently precise for the considered allowed transitions. An additional uncertainty lies in the evaluation of $g_{A}$, as nuclear models typically require (heavily) quenched values compared to the free-nucleon value [45]. Also in several shape factors reported here a dependence on $g_{A}$ is present. While our nuclear shell model results converge around $g_{A}^{\text {eff }} \simeq 0.9[31,42-44]$, all shape factors have been calculated within both a fully correlated and uncorrelated window for $g_{A} \in[0.7,1.27]$, taking the extremal values per bin as a $1 \sigma$ uncertainty [31]. Weak magnetism effects in allowed transitions are, however, calculated according to the benchmark analyses [2,3], which allows us to clearly separate the effect of forbidden transitions and directly compare against previously published results.

While several compilations have been produced in the past [25,55], forbidden decays have typically been pushed to the background as they only make up about $30 \%$ of the total number of transitions contributing to the total flux. Many of the large-endpoint transitions are, however, of forbidden nature due to the parity change of proton and neutron orbitals in the neutron-rich fission fragments. States of equal parity typically reside at excitation energies of several $\mathrm{MeV}$ with fragmented branching ratios, thereby pushing them out of the region of interest. To clarify these concerns, Fig. 3 shows the constituents of the summed full ${ }^{235} \mathrm{U}$ spectrum. It is immediately clear that allowed transitions, contrary to simple estimates, contribute less than $50 \%$ in the entire experimentally interesting region. In the observed shoulder, in particular, forbidden transitions constitute more than $60 \%$ of the total electron flux. The 


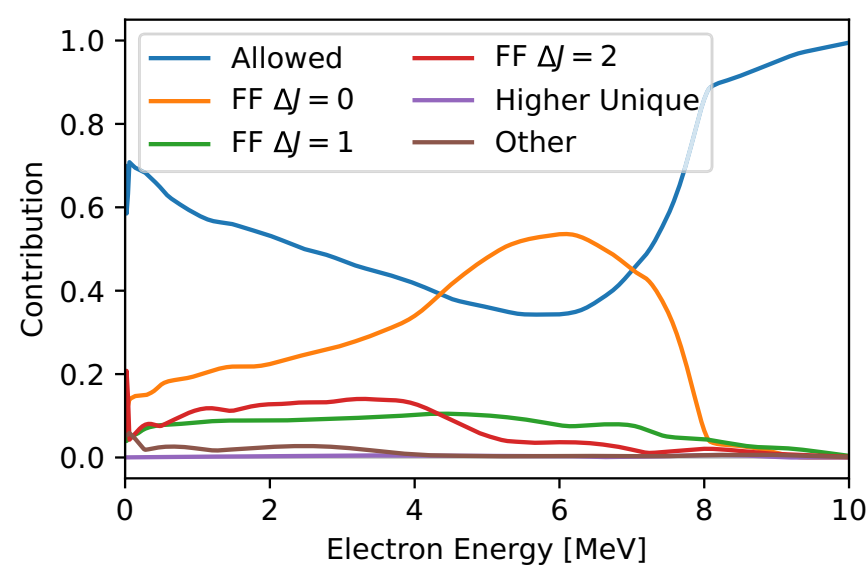

FIG. 3. Constituents of the summed ${ }^{235} \mathrm{U}$ electron spectrum using the ENDF database [48]. Here FF stands for first-forbidden and Other for non-unique transitions with $\Delta J \geqslant 2$. Behavior past $10 \mathrm{MeV}$ is dominated by a low number of branches. Using decay information from ENSDF (not shown here) these features are strongly amplified, with the contribution of allowed decays reaching a minimum below $20 \%$ around $5 \mathrm{MeV}$ [31].

majority of these are pseudoscalar transitions, which in a pure $0^{-} \leftrightarrow 0^{+}$transition show minimal deviation from an allowed equivalent. As shown above, however, this situation is not typical and subject to large higher-order contributions. Contributions from $\Delta J=1,2$ first-forbidden decays remain relatively constant throughout the entire spectrum up to $7 \mathrm{MeV}$, making up around $20 \%$. Given their strongly deviating shape factor as shown in Fig. 1, their influence cannot be neglected.

In light of these results and the relatively uniform behavior of the shape factors as calculated by the nuclear shell model, we attempt a simple parametrization. While the shape factor of pure pseudoscalar transitions [Eq. (3)] is simple enough, the influence of higher-order operators prevents a physically insightful function description. As such, we simply fit the obtained shape factors with a general description as in Eq. (4) and analogously for pseudovector transitions. The shape factor of unique forbidden decays [Eq. (2)] describes observed spectra within a few percent, which is sufficient for our purpose.

The parametrization then functions as follows [31]. Each of the non-unique shape factors calculated by the nuclear shell model is fit using functions described above. For each spin change $(\Delta J=0,1)$, one obtains distributions of fit parameters. Their spread is dominated by differences between transitions rather than individual uncertainties arising from $g_{A}$ and $\epsilon_{\mathrm{MEC}}$ ambiguity. Due to limited statistics, we use Gaussian kernel smoothing [56] where we manually set the bandwidth to $h=2$. Our choice results in fit parameter distributions with conservative uncertainties where all shape factors of Fig. 1 are contained within $\mathrm{a}<2 \sigma$ window. Full spectra are then calculated in a Monte Carlo fashion, where for each nonunique first-forbidden transition, fit parameters are obtained from the correlated parameter ensemble, with the exception of the transitions numerically calculated in this work. Repeating this procedure many times results in a translation of the

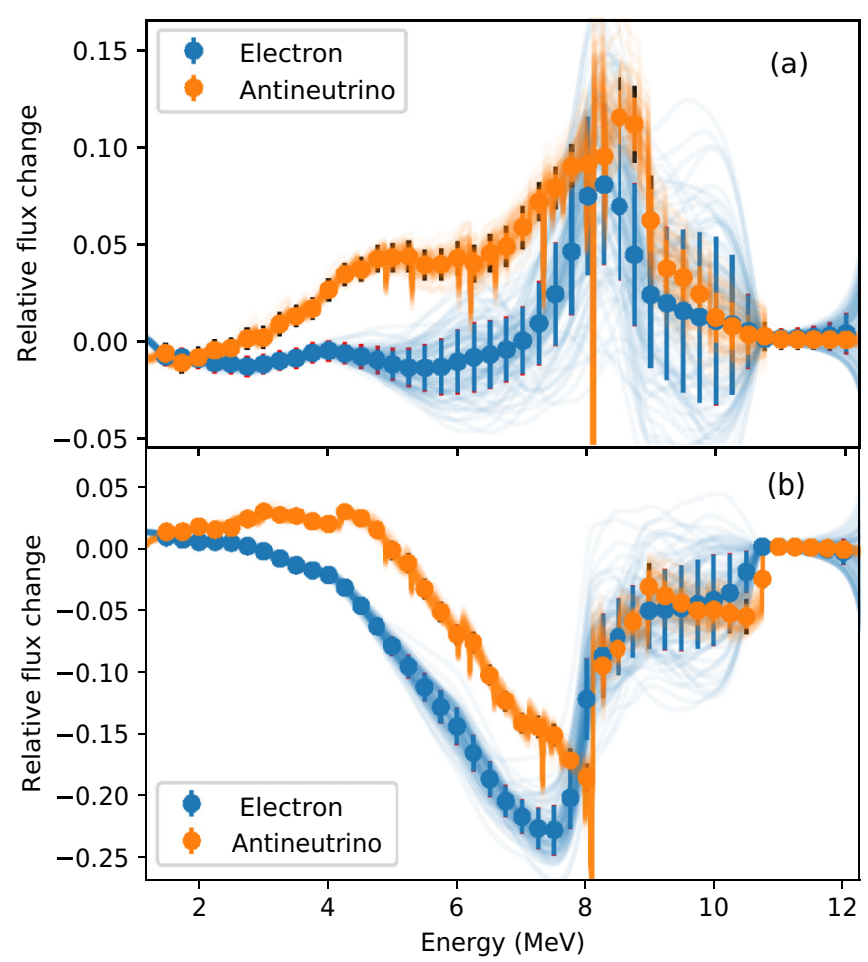

FIG. 4. Spectral change for electron and antineutrino cumulative spectra in the pure summation approach using the forbidden parametrization. The energy axis shows the kinetic energy of the electron and antineutrino. Top panel: Comparison against the allowed transition with a weak magnetism term. Bottom panel: Comparison against treating all forbidden decays as unique. Uncertainties result from a Monte Carlo calculation of 100 samples, together with a theory uncertainty of $1 \%$ from the uncertainty in the axial vector coupling constant $g_{A}$ and pseudoscalar mesonic enhancement [31].

parametrization uncertainty into a spectral uncertainty. The additional spectrum shape corrections in Eq. (1) are calculated using Ref. [57].

Figure 4 shows the spectral change and associated uncertainty for ${ }^{235} \mathrm{U}$ in the summation approach using 100 samples. We have made the comparison against the allowed approximation and against the Huber-Mueller method where all forbidden decays are treated as unique. We discuss both in turn.

As shown in Fig. 2, spectral changes to the electron cumulative spectrum are limited relative to the allowed approximation. The change in the antineutrino cumulative spectrum, on the other hand, shows significant deviations in the entire region of interest. Differences reach $5 \%$ in the 5-6 MeV region, showing an increase of the predicted neutrino flux relative to the allowed approximation. The uncertainty shown is an uncorrelated combination of a theory uncertainty of $1 \%$ due to the quenching of $g_{A}$ and mesonic corrections $[31,42]$ and the Monte Carlo uncertainty. Compared to treating all forbidden decays as unique, on the other hand, significant deviations in both electron and antineutrino cumulative spectra are observed. Considering the large differences in shape (shown in Fig. 1) this is hardly surprising. This will be 


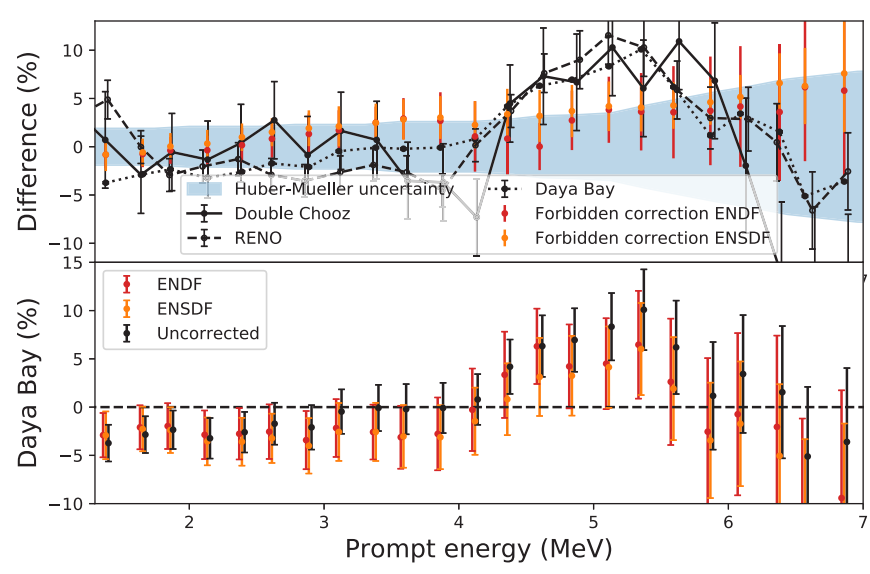

FIG. 5. Top panel: Normalized spectral ratios for three modern experiments relative to the Huber-Mueller predictions [2], and the normalized forbidden spectrum correction described in this work using ENDF and ENSDF decay libraries. The prompt energy of the positron emerging from the inverse $\beta$ decay is related to the antineutrino energy via $E_{\text {prompt }} \approx E_{v}-0.782 \mathrm{MeV}$. Bottom panel: Difference between Daya Bay spectral data and different theoretical models. Error bars are calculated using experimental, H-M, and forbidden uncertainties and are assumed uncorrelated. Here Uncorrected is relative to the $\mathrm{H}-\mathrm{M}$ estimate shown in the top panel, and ENDF and ENSDF are the new results.

the subject of further research with relation to the reactor normalization anomaly.

The starting point of the usual anomaly and spectral shoulder analysis is congruity with the ILL data. To guarantee this agreement, we employ a mixed summation+conversion method as in Ref. [2]. Differences in calculated electron spectra from the summation component using our different approximations are then compensated by the conversion part of the procedure. Aside from ${ }^{235} \mathrm{U}$ and ${ }^{238} \mathrm{U}$, however, summation predictions overestimate the experimental ILL data. For the ${ }^{239,241} \mathrm{Pu}$ isotopes, then, the reference electron spectra are set to the summation calculation in the allowed approximation. As the implementation of forbidden transitions lowers the expected electron flux (see Fig. 2), this introduced deficit can be recovered analogously with the conversion procedure [31]. The agreement with calculated and reference electron spectra is better than $1 \%$ up to $7 \mathrm{MeV}$, after which the uncertainty in the calculated antineutrino spectra is linearly increased with the observed discrepancy in electron spectra. By enforcing equivalence between electron spectra in our different approaches, the resultant antineutrino spectral changes can be directly compared to the experimentally observed shoulder.

Figure 5 shows the spectral ratios of Daya Bay [15], RENO [17], and Double Chooz [16] data relative to the HuberMueller prediction with the uncertainty of the latter. Additionally, we show the correction from forbidden transitions as described above using a normalized spectrum between 2 and $8 \mathrm{MeV}$ using the Daya Bay reactor composition [58], as is done for the experimental results. Further, we show the discrepancy of the Daya Bay spectral data with respect to our new calculations. The partial mitigation of the spectral shoulder and increased uncertainties arising from the treatment of first-forbidden transitions hint at a reduction in the statistical significance. The original spectral shoulder appears now to be compatible with theoretical estimates seeing how nearly all points agree within $1 \sigma$. Strong bin-to-bin correlations are present, however, to be discussed in a follow-up work [31].

In summary, we have for the first time performed microscopic calculations of the dominant forbidden transitions in the electron and antineutrino reactor spectra above $4 \mathrm{MeV}$. Through the use of a complete theoretical formalism, Coulomb corrections were taken into account at the appropriate level and shape factors strongly deviating from the usual allowed approximation were found. Using fission yield information, large changes were observed in the antineutrino spectrum. It was shown that despite being limited in number, forbidden transitions are the dominant component of the electron flux between 2 and $7 \mathrm{MeV}$. Based on the uniform behavior in the calculated shape factors, a parametrization of non-unique first-forbidden transitions was attempted. Using Monte Carlo methods, a spectral correction was obtained for all first-forbidden and higher uniquely forbidden transitions with an associated uncertainty. When compared to spectral discrepancies reported by all modern reactor neutrino experiments, the correction was shown to be of similar shape and magnitude. Taking these results at face value, a large portion of the reactor shoulder appears to be mitigated. Due to increased theoretical uncertainties arising from an improved treatment of first-forbidden transitions, the remaining spectral differences are of decreased statistical significance. Therefore, forbidden decays are not only non-negligible, but also an essential ingredient in the understanding of reactor antineutrino spectra and they merit additional research.
[1] G. Mention, M. Fechner, T. Lasserre, T. A. Mueller, D. Lhuillier, M. Cribier, and A. Letourneau, Phys. Rev. D 83, 073006 (2011).

[2] T. A. Mueller, D. Lhuillier, M. Fallot, A. Letourneau, S. Cormon, M. Fechner, L. Giot, T. Lasserre, J. Martino, G. Mention, A. Porta, and F. Yermia, Phys. Rev. C 83, 054615 (2011).

[3] P. Huber, Phys. Rev. C 84, 24617 (2011). 85, 029901(E) (2012).

[4] C. Athanassopoulos et al. (LSND Collaboration), Phys. Rev. Lett. 81, 1774 (1998).
[5] J. M. Conrad, W. C. Louis, and M. H. Shaevitz, Annu. Rev. Nucl. Part. Sci. 63, 45 (2013).

[6] F. Kaether, W. Hampel, G. Heusser, J. Kiko, and T. Kirsten, Phys. Lett. B 685, 47 (2010).

[7] K. N. Abazajian et al., arXiv:1204.5379.

[8] S. Gariazzo, C. Giunti, M. Laveder, Y. F. Li, and E. M. Zavanin, J. Phys. G: Nucl. Part. Phys. 43, 033001 (2015).

[9] Y. J. Ko et al. (NEOS Collaboration), Phys. Rev. Lett. 118, 121802 (2017). 
[10] J. Ashenfelter et al. (PROSPECT Collaboration), Phys. Rev. Lett. 121, 251802 (2018).

[11] I. Alekseev et al. (DANSS Collaboration), Phys. Lett. B 787, 56 (2018).

[12] H. Almazán et al. (STEREO Collaboration), Phys. Rev. Lett. 121, 161801 (2018).

[13] A. P. Serebrov et al. (Neutrino-4 Collaboration), arXiv:1805.10561.

[14] Y. Abreu et al. (SoLid Collaboration), J. Instrum. (2017) P04024.

[15] F. P. An et al. (Daya Bay Collaboration), Phys. Rev. Lett. 116, 061801 (2016).

[16] Y. Abe et al. (Double Chooz Collaboration), J. High Energy Phys. 10 (2014) 086.

[17] S. H. Seo et al. (RENO Collaboration), Phys. Rev. D 98, 012002 (2018).

[18] G. Mention, M. Vivier, J. Gaffiot, T. Lasserre, A. Letourneau, and T. Materna, Phys. Lett. B 773, 307 (2017).

[19] A. C. Hayes and P. Vogel, Annu. Rev. Nucl. Part. Sci. 66, 219 (2016).

[20] A. C. Hayes, J. L. Friar, G. T. Garvey, D. Ibeling, G. Jungman, T. Kawano, and R. W. Mills, Phys. Rev. D 92, 033015 (2015).

[21] P. Huber, Phys. Rev. Lett. 118, 042502 (2017).

[22] C. Buck, A. P. Collin, J. Haser, and M. Lindner, Phys. Lett. B 765, 159 (2017).

[23] A. C. Hayes, J. L. Friar, G. T. Garvey, G. Jungman, and G. Jonkmans, Phys. Rev. Lett. 112, 202501 (2014).

[24] D.-L. Fang and B. A. Brown, Phys. Rev. C 91, 025503 (2015).

[25] A. A. Sonzogni, T. D. Johnson, and E. A. McCutchan, Phys. Rev. C 91, 011301 (2015).

[26] H. Behrens and W. Bühring, Electron Radial Wave Functions and Nuclear Beta-Decay (Clarendon Press, Oxford, 1982).

[27] L. Hayen, N. Severijns, K. Bodek, D. Rozpedzik, and X. Mougeot, Rev. Mod. Phys. 90, 015008 (2018).

[28] D. A. Dwyer and T. J. Langford, Phys. Rev. Lett. 114, 012502 (2015).

[29] H. Behrens and J. Jänecke, Landolt-Börnstein Tables, Gruppe I, Band 4 (Springer, Berlin, 1969).

[30] H. Behrens and W. Bühring, Nucl. Phys. A 162, 111 (1971).

[31] L. Hayen, J. Kostensalo, N. Severijns, and J. Suhonen (unpublished).

[32] K. Schreckenbach, H. Faust, F. von Feilitzsch, A. Hahn, K. Hawerkamp, and J. Vuilleumier, Phys. Lett. B 99, 251 (1981).
[33] F. V. Feilitzsch, A. Hahn, and K. Schreckenbach, Phys. Lett. B 118, 162 (1982).

[34] K. Schreckenbach, G. Colvin, W. Gelletly, and F. Von Feilitzsch, Phys. Lett. B 160, 325 (1985).

[35] A. A. Hahn, K. Schreckenbach, W. Gelletly, F. von Feilitzsch, G. Colvin, and B. Krusche, Phys. Lett. B 218, 365 (1989).

[36] N. Haag, W. Gelletly, F. von Feilitzsch, L. Oberauer, W. Potzel, K. Schreckenbach, and A. A. Sonzogni, arXiv:1405.3501.

[37] B. A. Brown and W. D. M. Rae, Nucl. Data Sheets 120, 115 (2014).

[38] H. Mach, E. K. Warburton, R. L. Gill, R. F. Casten, J. A. Becker, B. A. Brown, and J. A. Winger, Phys. Rev. C 41, 226 (1990).

[39] R. Machleidt, Phys. Rev. C 63, 024001 (2001).

[40] S. Lalkovski et al., Phys. Rev. C 87, 034308 (2013).

[41] B. A. Brown (unpublished).

[42] J. Kostensalo and J. Suhonen, Phys. Lett. B 781, 480 (2018).

[43] J. Kostensalo, M. Haaranen, and J. Suhonen, Phys. Rev. C 95, 044313 (2017).

[44] M. Haaranen, J. Kotila, and J. Suhonen, Phys. Rev. C 95, 024327 (2017).

[45] J. Suhonen, Front. Phys. 5, 55 (2017).

[46] L. Bodenstein-Dresler et al. (COBRA Collaboration), arXiv: 1806.02254.

[47] "Evaluated nuclear structure data file", https://www.nndc.bnl. gov/ensdf/ (2018).

[48] M. Chadwick et al., Nucl. Data Sheets 112, 2887 (2011).

[49] J. C. Hardy, L. C. Carraz, B. Jonson, and P. G. Hansen, Phys. Lett. B 71, 307 (1977).

[50] I. Gauld, M. Pigni, and G. Ilas, Nucl. Data Sheets 120, 33 (2014).

[51] E. Mendoza, F. Álvarez-Velarde, V. Bécares, D. CanoOtt, E. González-Romero, T. Martínez, and D. Villamarín, Nucl. Instrum. Methods Phys. Res. Sect. A 870, 60 (2017).

[52] B. Holstein, Rev. Mod. Phys. 46, 789 (1974).

[53] N. Severijns, L. Hayen, and I. Towner (unpublished).

[54] X. B. Wang and A. C. Hayes, Phys. Rev. C 95, 064313 (2017).

[55] A. C. Hayes, G. Jungman, E. A. McCutchan, A. A. Sonzogni, G. T. Garvey, and X. B. Wang, Phys. Rev. Lett. 120, 022503 (2018).

[56] D. W. Scott, Multivariate Density Estimation: Theory, Practice, and Visualization (Wiley, New York, 1992).

[57] L. Hayen and N. Severijns, arXiv:1803.00525.

[58] F. P. An et al. (Daya Bay Collaboration), Phys. Rev. Lett. 108, 171803 (2012). 